\title{
An application and Parallel Tabu Search Algorithm for Solving the PTSP Under the OpenMP-MPI Environment
}

\author{
Mohamed Abdellahi Amar ${ }^{* 1}$ and Walid Khaznaji ${ }^{2}$ \\ ${ }^{1}$ CRISTAL-GRIFT Laboratory, National School of Computer Science, University of Manouba \\ Manouba, Tunisia \\ medabdellahiamareyahoo.fr \\ ${ }^{2}$ Tunisia SESAME University, \\ Ariana,Tunisia \\ walid.khaznaji@sesame.com.tn
}

\begin{abstract}
This paper reviews some real-world problems modeling as Probabilistic Traveling Salesman Problem (PTSP), by presenting the important results found in the literature. It illustrates the usefulness of the inclusion of probabilistic elements in deterministic models. We propose a new modeling of the PTSP by the deviations of the routing of a robot in order to avoid obstacles which are not foreseen in its path. The Probabilistic Traveling Salesman Problem(PTSP) is a variation of the classic Traveling Salesman Problem (TSP) where each node $i$ is present with probability $p_{i}$. The solution for the PTSP consists in finding an a priori tour that visits all the cities that minimizes the expected length of the tour. From the litterateur the PTSP is NP-Complete, therefore the execution time is a prime factor in its resolution. In the last of his paper we present a new parallel Tabu search heuristic for solving PTSP by using the Open MPI environment.
\end{abstract}

\section{INTRODUCTION}

There are various motivations for studying the effect of including probabilistic elements in combinatorial optimization problems COP. The reason for this is that many of the COPs have concrete applications. For example, for many delivery companies, only a subset of their customers requires a delivery each day. The most natural approach that comes in mind is to consider each potential instance as a new problem defined through the present data and to optimally solve the instance considered. This approach is called re-optimization strategy. This approach is optimal, however, it can be very much time and space consuming, in particular when the COP considered is NP-complete [1], [2], [3]. It is therefore necessary to adopt another resolution strategy, which is less costly in terms of computations. This approach is called an a priori optimization and has been introduced in [1]. It consists of determining a solution of the initial instance, where all data are present, called an a priori solution, and applying a strategy called a modification strategy to adapt as quickly as possible the a priori solution to the sub-instance that must effectively be solved.
The Probabilistic Traveling Salesman Problem (PTSP) is a probabilistic extension of the well-known Traveling Salesman Problem (TSP) was introduced by Jaillet [1] where it assumes that the number of cities is a random variable, that is the cities of TSP may or may not be present according to a probability of presence. The inclusion of probabilistic elements in TSP admit an immediate interest, which is the modeling of practical problems for which randomness is a constant source of preoccupation, also it can be used to model many realworld applications [1], [4]. Bertsimas and al. [2] showed that the PTSP is a NP-Complete problem, and it's harder than its deterministic version. Therefore many approximate methods were proposed to solve the PTSP. We cite, stochastic simulated annealing [5], genetic algorithm [6], ant colony system [7], and other [8]. For literature on the PTSP, a very limited number of papers have seen the light for the parallelization of the PTSP [9], [10], [11], [12] Our goal is to propose a parallel Tabu search heuristic (TS) for the PTSP, by using the methods of local search heuristics 2-opt. In this context, the strategy consists in studying the parallelization of the phenomena of intensification and diversification of the TS.

The paper is organized as follows: We begin in section 2 by the definition of the PTSP and its formulation. In section 3 we give a view on modeling. In section 4 we present the models of deviations of the routing of a robot as PTSP. Then in section 5 we introduce the definition of Tabu search and local search 2-opt. In section 6 we present the numerical results. Finally, section 7 contains the conclusion and future work.

\section{PROBABILISTIC TRAVELING SALESMAN PROBLEM}

The Probabilistic Traveling Salesman Problem (PTSP) is an extension of a variation of the classical Traveling Salesman Problem (TSP). It can be formulated as follows [1]: let a fully connected graph whose set of nodes is denoted by $V=\{1,2,,,,, n\}$. Given an a priori tour $\mathrm{T}$, and the subset of $S: P(V)$ that will occur with probability $\mathrm{P}(\mathrm{S})$. Let $L_{T}(S)$ be a length of instance $S$. The resolution of the problem is 
to find an a priori tour through all $n$ potential nodes, which minimizes the quantity: 1 [1].

$$
\min _{T} E\left(L_{T}\right)=\sum_{S \subseteq V} P(S) L_{T}(S)
$$

Let $E$ be a set of edges $E=\{(i, j), 0 \leq i \leq n, 0 \leq j \leq n\}$. To each edge $(i, j)$ is associated a distance $\mathrm{d}_{i j}$.

The expression of objective function was introduced by Jaillet [1]. Let $T=(1,2, \ldots, n, 1)$ an a priori tour and each vertex has a probability of presence $p_{i}$

$$
\begin{aligned}
& E\left(L_{T}\right)=\sum_{i=1}^{n} \sum_{j=i+1}^{n} d_{i j} p_{i} p_{j} \prod_{k=i+1}^{j-1}\left(1-p_{k}\right) \\
& +\sum_{i=1}^{n} \sum_{j=1}^{i-1} d_{i j} p_{i} p_{j} \prod_{k=i+1}^{n}\left(1-p_{k}\right) \prod_{l=1}^{j-1}\left(1-p_{l}\right)
\end{aligned}
$$

In the case when the $p_{i}=p(q=1-p) \forall i$, according to [1], the expected length of an a priori tour $T=(1,2, \ldots, n, 1)$ becomes

$$
E\left(L_{T}\right)=p^{2} \sum_{r=0}^{n-2} q^{r} \sum_{i=1}^{n} d\left(i, T^{r}(i)\right)
$$

Where $T^{r}(i)$ is the successor number $\mathrm{r}$ of $i$ in the tour $T$. Other paper discus this objective function of the PTSP, for example in [5] the objective function of the PTSP is estimated by sampling.

\section{A VIEW ON MODELING}

This first problem was introduced by Jaillet [1], who studied some of its properties and derived asymptotic results. Vast various papers were proposed different heuristics [1] [5], [13]. Also many papers discuss the modeling of this problem by concrete application. The authors in [4] use of this a priori strategy allows the wireless sensor network to collect effectively detected information from external environments and deliver it to the required applications with reducing energy consumption, even if some nodes are destroyed. Concerning the problem of optimization of the routing path at KAT-mobility, the authors in [4] proposed that only certain group centers among the $K$ vertices actually require a visit according to their probabilities of presence. In other words, they applied the strategy a priori they got a tour through the $K$ initial vertices such that the new tour through the present vertices is obtained from the a priori tour $T$ according to the modification method $\mathcal{U}$. This is the problem of finding the tour that minimizes the expected distance traveled [1]. So one has a probability distribution $P$, the set of group centers, the set of all subsets of $V$, i.e. each exemplary $V^{\prime} \subseteq V$ has a probability of presence $P\left(V^{\prime}\right)$. Let
$R$ be a tour through the centers, The method $\mathcal{U}$ consists to erase the absent centers of the a priori tour. Let $L(R, \mathcal{U})$ the random variable, that for all $V^{\prime} \subseteq V$ and has a priori tour $R$, associate the length $L\left(R\left(V^{\prime}, \mathcal{U}\right)\right)$ of the tour, through $V^{\prime}$, by the method of modification $\mathcal{U}$ from $R$. Accordingly, the optimization of the path of the mobile collector in order to visit once and for all the center sensors of the groups is equivalent to searching for a tour which minimizes the expected of the random variable $L((R, \mathcal{U}))$

$$
\min _{R} E\left(L_{R}\right)=\sum_{S \subseteq V} P(S) L_{R, \mathcal{U}}(S)
$$

Other papers have been found in litterateur in order to model many real-world applications [1], [14], [15], [8].

\section{MOdeling ROUTING OF A ROBOT}

There are two main families of maps in the literature that allow connecting perception, decision and action: maps in the form of occupancy grids, which are a metric discretization of the environment and topological maps. They represent the environment in the form of a graph where the nodes correspond to particular locations and the arcs represent the connectivity between the nodes [16] [17], [18]. Knowing a map of the environment and the position of the robot within this map, it is possible to calculate a trajectory to reach a goal. We consider robots capable of moving in a 2-dimensional space whose commands influence with simple relationships on the position in this space. The calculation of the displacements can therefore be done directly in the space of the map. Planning algorithms generally use path search methods in graphs. It is therefore necessary to represent the map in the form of a graph. The topological maps directly provide this graph.

\section{A. Within a topological map}

Robots are transported in a wide variety of environments that can be grouped into two main categories: static environments and dynamic environments. Static environments are environments that do not undergo changes over time. This stability concerns both their spatial structure and their appearance for the sensors of the robot [17]. This excludes the majority of environments in which humans daily evolve. Dynamic environments, on the other hand, exhibit characteristics that evolve over time. Most common environments obviously belong to the second category. For example, an office environment is dynamic, because people who work there, chairs that are moved or doors that are open or closed. This uncertainty is characteristic of dynamic environments, in which objects are likely to move, appear or disappear. Since that the topological map reproduces the raw data perceived by the sensors of the robot. So we find ourselves in the framework of PTSP and the Probabilistic Shortest Path Problem (PSPP) according to whether the path is closed or not as shown in figures 1 and 2 . 


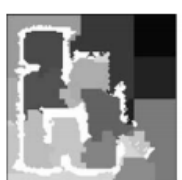

(a)

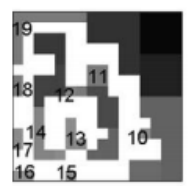

(e)

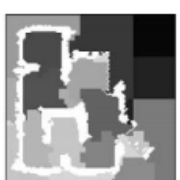

(b)

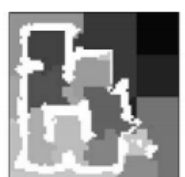

(c)

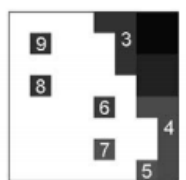

(f)

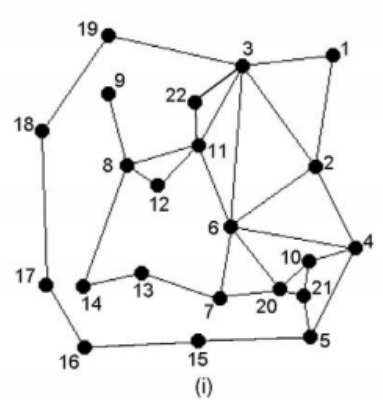

Fig. 1. Topological map extraction: (a) base level; (b) level 1; (c) level 2; (d) level 3 yielding three topological nodes; (e) level 4 yielding 10 topological nodes; (f) level 5 yielding seven topological nodes; (g) level 6 yielding two topological nodes; (h) level 7; (i) topological map [19].
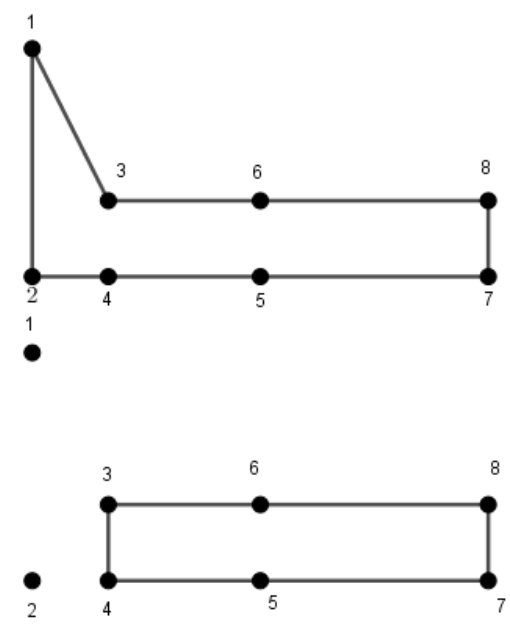

Fig. 2. An a priori tour through 8 points (up), and the sub-tour solution when the points 1,2 are absent, utilizing modification method $\mu$, by keeping the same order (below).

\section{B. Within an occupancy grid mapping}

Obstacle avoidance is a basic behavior found in virtually all mobile robots [17]. It is essential to enable the robot to operate in a dynamic environment and to manage the gaps between the internal model and the real world. As that occupancy grids represent the environment in which space is discretized into regular cells and each cell has an associated probability of being occupied by an obstacle. It is clear that the problem of avoiding obstacles in this context belongs to a PTSP or PSPP depending on whether the path is closed or not.

\section{TABU SEARCH HEURISTIC (TS)}

We consider a graph $G=(V, E)$, where $V=\{1, \ldots \ldots, n\}$ is a set of vertexes and $E$ is the set of edges $E=\{(i, j), 0 \leq$ $i \leq n, 0 \leq j \leq n\}$. To each edge $(i, j)$ is associated a distance $\mathrm{d}_{i j}$.

The expression of objective function was introduced by Jaillet [1]. Our resolution is limited to the homogeneous PTSP. Let $T=(1,2, \ldots, n, 1)$ be an a priori tour and each vertex has a probability of presence $p=p_{i}$, the expected cost of $\mathrm{T}$ is shown in formula $\mathrm{V}$

$$
E\left(L_{T}\right)=p^{2} \sum_{r=0}^{n-2} q^{r} \sum_{i=1}^{n} d\left(i, T^{r}(i)\right)
$$

Where $T^{r}(i)$ is the successor number $\mathrm{r}$ of $i$ in the tour $T$. The Tabu heuristic was developed by Glover [20], it consists of a set of general rules and mechanisms. As a function of controlling and guiding an internal heuristic specifically adapted to the problem to be solved, in order to allow it to transcend the obstacle of the local optima. Unlike genetic algorithms or annealing simulated that use probabilistic arguments to achieve this objective. The TS exploits the notion of memory. Very few papers of TS were proposed to solve the PTSP. [21].

\begin{tabular}{|c|c|}
\hline Tabu list & Stopping criterion \\
\hline Size of problem & Would be to stop after a given number of iterations \\
\hline
\end{tabular}

TABLE I

PARAMETERS OF THE TABU HEURISTIC

Local search 2-opt which consists in choosing two nonadjacent edges in the Hamiltonian cycle and replacing them by the two edges which make it possible to reconstitute a Hamiltonian cycle [7]. For calculating the difference of the expected length of tour $T(1,2, \ldots n, 1)$, which we denote $\Delta_{i j}$, we introduce for its computation two matrices $A$ and $B$ defined as follows: Let $j=i+k$ is the $k^{t h}$ successor of $i$ in $T$

$$
A_{i, k}=\sum_{r=k}^{n-1} q^{r-1} d(i, i+r), B_{i, k}=\sum_{r=k}^{n-1} q^{r-1} d(i-r, i)
$$

with $1 \leq k \leq n-1$ and $1 \leq i \leq n$ the calculation of $\Delta_{i j}$ is as follows if $k=1$ we have

$$
\begin{gathered}
\Delta_{i, i+1}=p^{3}\left[q^{-1} A_{i, 2}-\right. \\
\left.B_{i, 1}+B_{i, n-1}-A_{i+1,1}+A_{i+1, n-1}+q^{-1} B_{i+1,2}\right]
\end{gathered}
$$

And for all $k \geq 2$

$$
\begin{gathered}
\Delta_{i, j}=\Delta_{i+1, j-1}+p^{2}\left[\left(q^{-k}-1\right) A_{i, k+1}+\right. \\
\left(q^{k}-1\right)\left(B_{i, 1}-B_{i, n-k}\right)+\left(q^{k}-1\right)\left(A_{j, 1}-A_{j, n-k}\right)+ \\
\left(q^{-k}-1\right)\left(B_{j, k+1}\right)+\left(1-q^{n-k}\right)\left(A_{i, 1}-A_{i, k}\right) \\
+\left(1-q^{k-n}\right) B_{i, n-k+1}+\left(1-q^{n-k}\right) \\
\left.\left(B_{j, 1}-B_{j, k}\right)+\left(1-q^{k-n}\right) A_{j, n-k+1}\right]
\end{gathered}
$$




\section{NUMERICAL RESULTS}

We used the programming language $\mathrm{C}$ and the OpenMP library for the parallel implementation. We implemented the TS with the 2-opt structure, on a machine whose characteristics are: Windows 7 System: Processor: Intel Core (2) 2 Quad CPU Q8200@2.33GHZ2.33 GHZ Ram: 4.00 GB (3.44 GB usable). System type: 32-bit OS. According to the sequential study of the TS for the PTSP, we have noticed that computing time remains a major disadvantage especially when the number of cities becomes important. For this purpose we propose a new strategy of parallelization of the TS. In this experimental part we will implement it of this method which is the parallelization $1 \Delta_{i, j}$. It is the cost difference between a tour and its neighbor, obtained by a 2-opt transformation.

\begin{tabular}{c|c|cccc}
\hline & & \multicolumn{3}{|c}{2 proc } \\
\hline $\mathrm{p}$ & $\mathrm{N}$ & Tseq & Tparal & speedup & Eff \\
\hline 0.1 & 50 & 0.025 & 0.022 & 1.136 & \\
& 100 & 0.733 & 0.579 & 1.265 & 0.632 \\
& 150 & 4.527 & 4.558 & 1.015 & 0.507 \\
& 200 & 32.47 & 20.49 & 1.584 & 0.792 \\
\hline 0.7 & 50 & 0.018 & 0.016 & 1.125 & 0.562 \\
& 100 & 0.732 & 0.676 & 1.082 & 0.541 \\
& 150 & 5.124 & 4.856 & 1.055 & 0.527 \\
& 200 & 20.03 & 17.15 & 1.167 & 0.583 \\
\hline 0.9 & 50 & 0.019 & 0.017 & 1.117 & 0.558 \\
& 100 & 0.679 & 0.600 & 1.131 & 0.565 \\
& 150 & 5.430 & 5.259 & 1.032 & 0.516 \\
& 200 & 24.47 & 21.75 & 1.125 & 0.562
\end{tabular}

\section{TABLE II}

SPEEDUP $=\frac{T \text { seq }}{\text { Tparal }}$ AND EFFICIENCY $(\mathrm{EFF})=\frac{\text { Speedup }}{n p}$ FOR 2 AND 4 PROCESSORS(PROC) AND P $=0.1,0.7$ AND 0.9 (TSEC $=$ SEQUENTIAL TIME, TPARAL $=$ PARALLEL TIME, NB IS NUMBER OF PROCESSORS)

\begin{tabular}{c|c|cccc}
\hline & & \multicolumn{4}{|c}{ 4 proc } \\
\hline $\mathrm{p}$ & $\mathrm{N}$ & Tseq & Tparal & speedup & Eff \\
\hline 0.1 & 50 & 0.025 & 0.014 & 1.785 & 0.446 \\
& 100 & 0.733 & 0.383 & 1.913 & 0.478 \\
& 150 & 4.527 & 2.140 & 2.115 & 0.528 \\
& 200 & 32.47 & 16.020 & 2.026 & 0.506 \\
\hline 0.7 & 50 & 0.018 & 0.010 & 1.800 & 0.450 \\
& 100 & 0.732 & 0.348 & 2.103 & 0.525 \\
& 150 & 5.124 & 2.147 & 1.920 & 0.480 \\
& 200 & 20.03 & 10.010 & 2.00 & 0.500 \\
\hline 0.9 & 50 & 0.019 & 0.010 & 1.900 & 0.475 \\
& 100 & 0.679 & 0.338 & 2.008 & 0.502 \\
& 150 & 5.430 & 2.6770 & 2.028 & 0.507 \\
& 200 & 24.47 & 12.160 & 2.012 & 0.503
\end{tabular}

\section{TABLE III}

SPEEDUP $=\frac{T \text { seq }}{\text { Tparal }}$ AND EFFICIENCY $($ EFF $)=\frac{\text { Speedup }}{n p}$ FOR 2 AND 4 PROCESSORS(PROC) AND P $=0.1,0.7$ AND 0.9 (TSEC= SEQUENTIAL TIME,

TPARAL $=$ PARALLEL TIME, NB IS NUMBER OF PROCESSORS)

The Table III shows the speedup and efficiency obtained for different values of the number of $\mathrm{N}$ cities. The analysis of this table shows that speedups and efficiencies improve when $\mathrm{N}$ increases (for a fixed number of processors) and also with the number of processors (for a fixed number of $\mathrm{N}$ cities) and we have noticed that the execution time is much reduced, where the efficiency equals to 0.792 for $\mathrm{p}=0.1$. Figures 3 ,4 and 5 clearly show that there is good speedups and that

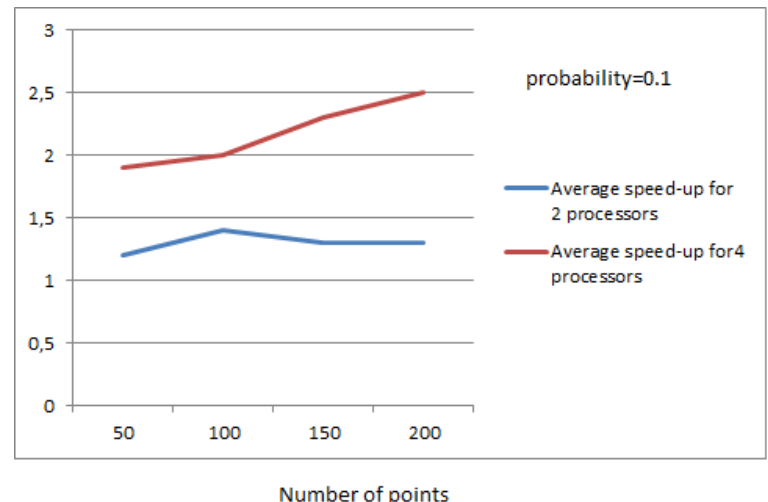

Fig. 3. Speedup as function of number of points for probability $\mathrm{p}=0.1$

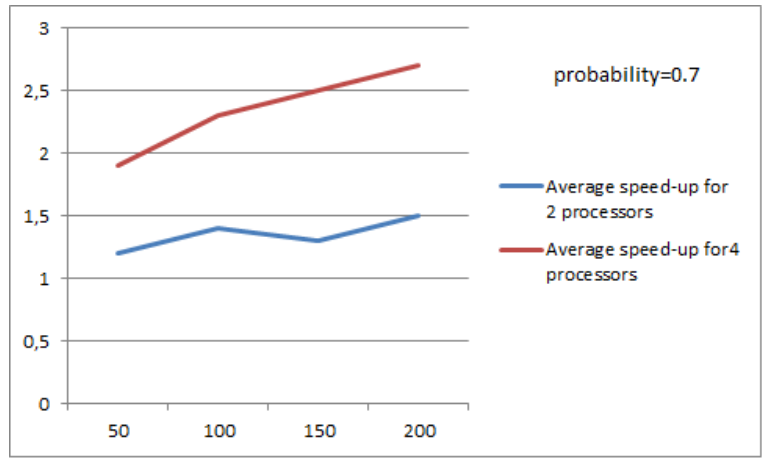

Number of points

Fig. 4. Speedup as function of number of points for probability $\mathrm{p}=0.7$

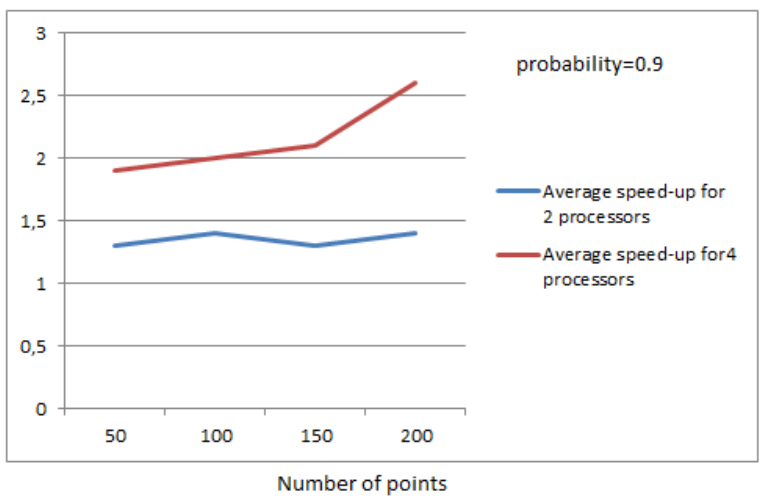

Fig. 5. Speedup as function of number of points for probability $\mathrm{p}=0.9$

speedup is an increasing function as a function of the number of processors and cities. This implies that the proposed strategy of parallelization of matrices A and B is very efficient.

\section{CONCLUSION AND FUTURE WORK}

In this paper, we were presented the notion of the PTSP and giving its motivation and formulation. It is very worthy that the probabilistic notion continues to be developed because its capacity to model real-world situations. We revealed the hidden links that exist between the notion of "map" used in mobile robotics and the PTSP, where the problem of avoiding 
obstacles directly represents the PTSP. We proposed a strategy of parallelization of Tabu search for solution of the PTSP. It evaluated the performance of the approach on a parallel machine. From the results obtained, we can conclude that the parallel version of the TS was well adapted for PTSP. As the sequential TS is very greedy in computing time, the use of parallel computers allows to accelerate their execution, it gave 2 as speedup. This was pointedly proved the effectiveness of this strategy of parallelization. Our future work includes a parallelization of the heuristic in a heterogeneous environment and on a grid massively using the MPI-OpenMP.

\section{REFERENCES}

[1] P. Jaillet, "The probabilistic traveling salesman problems," Technical report 185, Operations Research, MIT, Cambridge, Mass., 1985.

[2] D. Bertsimas and L. Howell, "Further results on the probabilistic traveling salesman problem," European Journal of Operational Research, vol. 65, pp. 68-95, 1993.

[3] M. Bellalouna, "Problèmes d'optimisation combinatoire probabilistes," Ph.D. dissertation, Ecole Nationale des Ponts et Chaussées, Paris, France, 1993.

[4] M. Bellalouna, A. Gharbi, and W. Khaznaji, "The k-means and tsp based mobility protocol modeling as a probabilistic combinatorial optimization problem," in The Ninth International Conference on Systems and Networks Communications, 2014, pp. 48-53.

[5] N. E. Bowler, T. M. A. Fink, and R. C. Ball, "Characterization of the probabilistic traveling salesman problem," Physical Review E, vol. 68, p. 036703, 2003.

[6] J. R.-C. Liu, Y-H. and C.-J. Wang, "Genetic algorithms for the probabilistic traveling salesman problem," in Lecture Notes in Artifical Intelligence, 2004, pp. 77-82.

[7] L. Bianchi, "Ant colony optimization and local search for the probabilistic traveling salesman problem: a case study in stochastic combinatorial optimization," Ph.D. dissertation, Univ. Libre de Bruxelles, Brussels, Belgium, 2006.

[8] A. Henchiri, M. Bellalouna, and W. Khaznaji, "A probabilistic traveling salesman problem: a survey," in The Federated Conference on Coputer Science and Information Systems FedCSIS, vol. 3, 2014, pp. 55-60.

[9] D. Weyland, R. Montemanni, and L. M. Gambardella, "Heuristics for the probabilistic traveling salesman problem with deadlines based on quasi-parallel monte carlo sampling," Computers \& operations research, vol. 40, no. 7 , pp. 1661-1670, 2013.

[10] D. Weyland, "On the computational complexity of the probabilistic traveling salesman problem with deadlines," Theoretical Computer Science, vol. 540, pp. 156-168, 2014.

[11] M. A. Amar, W. Khaznaji, and M. Bellalouna, "A parallel hybrid heuristic based on karp's partitioning for ptsp on multi-core processors,' in 2017 IEEE/ACIS 16th International Conference on Computer and Information Science (ICIS). IEEE, 2017, pp. 465-470.

[12] _ "A parallel branch and bound algorithm for the probabilistic tsp," in International Conference on Algorithms and Architectures for Paralle Processing. Springer, 2018, pp. 437-448.

[13] L. Bianchi, M. Dorigo, L. M. Gambardella, and W. J. Gutjahr, "A survey on metaheuristics for stochastic combinatorial optimization," Natural Computing, vol. 8, no. 2, pp. 239-287, 2009.

[14] D. Bertsimas, P. Chervi, and M. Peterson, "Computational approaches to stochastic vehicle routing problems," Transportation Science, vol. 4, pp. 342-352, 1995.

[15] A. Campbell, "Aggregation for the probabilistic traveling salesman problem," Computers \& Operations Research, vol. 33, 2703-2724, 2006

[16] R. Grasse, Y. Morère, and A. Pruski, "Assisted navigation for persons with reduced mobility: Path recognition through particle filtering (condensation algorithm)," Journal of Intelligent \& Robotic Systems, vol. 60, no. 1, pp. 19-57, 2010. [Online]. Available: http://dx.doi.org/10.1007/s10846-010-9406-y

[17] D. Filliat, "Robotique mobile," 2011.

[18] G. Lozenguez, L. Adouane, A. Beynier, A.-I. Mouaddib, and P. Martinet, "Calcul distribué de politiques d'exploration pour une flotte de robots mobiles," in Journées Francophone des Systèmes Multi-Agent, Valenciennes, France, 2011, pp. -.
[19] A. Poncela, E. J. Perez, A. Bandera, C. Urdiales, and F. Sandoval, "Efficient integration of metric and topological maps for directed exploration of unknown environments," Robotics and Autonomous Systems, vol. 41, no. 1, pp. 21-39, 2002.

[20] F. Glover, "Future paths for integer programming and links to artificial intelligence," Computers \& Operations Research, pp. 13, 533-549, 1986.

[21] Y. Marinakis and M. Marinaki, "A hybrid multi-swarm particle optimization algorithm for the probabilistic traveling salesman problem," Computers and Operations Research, vol. 37, pp. 432-442, 2010. 